\title{
VLA-4 as a Central Target for Modulating Neuroinflammatory Disorders
}

\author{
Arnon Dias Jurberg ${ }^{a, b, c, d}$ Beatriz Chaves ${ }^{b, e} \quad$ Lia Gonçalves Pinho ${ }^{a}$, b, c \\ João Hermínio Martins da Silvab, e Wilson Savino ${ }^{a, b, c}$ \\ Vinicius Cotta-de-Almeida ${ }^{a, b, c, d}$ \\ aLaboratory on Thymus Research, Oswaldo Cruz Institute, Oswaldo Cruz Foundation, Rio de Janeiro, Brazil; \\ ${ }^{b}$ National Institute of Science and Technology on Neuroimmunomodulation (INCT-NIM), Oswaldo Cruz Institute, \\ Oswaldo Cruz Foundation (Fiocruz), Rio de Janeiro, Brazil; 'Rio de Janeiro Research Network on Neuroinflammation \\ (RENEURIN), Oswaldo Cruz Institute, Oswaldo Cruz Foundation, Rio de Janeiro, Brazil; ${ }^{\text {d}}$ School of Medicine, Estácio \\ de Sá University, Rio de Janeiro, Brazil; ' ${ }^{C}$ Computational Modeling Group, Oswaldo Cruz Foundation (Fiocruz), \\ Eusébio, Brazil
}

\section{Keywords}

Integrin · Migration - T cells · Multiple sclerosis ·

Natalizumab

\section{Abstract}

The complex steps leading to the central nervous system (CNS) inflammation and the progress to neuroinflammatory and neurodegenerative disorders have opened up new research and intervention avenues. This review focuses on the therapeutic targeting of the VLA-4 integrin to discuss the clear-cut effect on immune cell trafficking into brain tissues. Besides, we explore the possibility that blocking VLA-4 may have a relevant impact on nonmigratory activities of immune cells, such as antigen presentation and T-cell differentiation, during the neuroinflammatory process. Lastly, the recent refinement of computational techniques is highlighted as a way to increase specificity and to reduce the detrimental side effects of VLA-4 immunotherapies aiming at developing better clinical interventions.

(c) 2021 S. Karger AG, Basel

karger@karger.com

(C) 2021 S. Karger AG, Basel

www.karger.com/nim

\section{Introduction}

A growing number of individuals diagnosed with neurodegenerative disorders have been observed in recent years, as the world population gets progressively older [ 1 , 2]. In particular, aging is recognized as a risk factor for many brain-related diseases, such as Alzheimer's disease and other dementia, or yet neuromotor-related pathologies, like Parkinson's disease and amyotrophic lateral sclerosis (ALS) $[3,4]$. This correlation likely arises from the progressive decline in the activities of overlapping mechanisms encompassing metabolic dysregulation, stress pathways, macromolecular damage, and defective proteostasis, as well as epigenetic changes and impaired regeneration of adult stem cells. Noteworthy, all these processes are underlined by an age-correlated gradual increase in the basal inflammatory status of the individuals, namely, inflammaging $[5,6]$. This scenario grows in complexity, as the existence of different types of aging patterns (or "ageotypes") have been proposed upon the identification of enriched pathways for immunity, metabolism, and inflammation, as well as liver and kidney dysfunctions [7]. Whether one specific pathway or a com-

\footnotetext{
Karger"
} 
bination of 2 or more pathways is involved in the emergence of neurodegeneration remains to be further elucidated. Nevertheless, an examination of these findings revealed that many cell adhesion molecules associated with leukocyte trafficking are positively or inversely correlated with age.

The migrating inflammatory cells, in their pathway toward a given peripheral tissue, face particular interactions with both the endothelium and the subjacent tissue microenvironment. The pathway to access the target tissue finds the crossing of blood vessel as ordered steps of tethering, rolling, firm adhesion, and transmigration governed by critical adhesion molecules and chemokines [8]. This raises a tantalizing possibility that targeting cell adhesion molecules can produce new opportunities to halt prematurely the low-grade inflammation and delay neurodegenerative progression. Remarkably, the integrin Very Late Antigen-4 (VLA-4; $\alpha 4 \beta 1$; CD49d/CD29) is regarded as a pivotal molecule regulating the adhesive phase of the transmigratory process and it has been a therapeutic target in inflammatory diseases [9]. In this review, we explored the VLA-4 molecule as a target of intervention for neuroinflammatory disorders.

\section{VLA-4-Driven Immune Cell Trafficking toward the Inflamed Brain}

The immune cells and their mediators are part of a complex homeostatic system responsible to monitor the entire body. The particular anatomy and organization of the central nervous system (CNS) have fueled a view of immune privilege, as discussed elsewhere [10-12]. Notwithstanding, some immune cell types play nondefensive roles in the brain and their mechanisms of action have only begun to be unraveled. While microglia have classically stood out as the watchman of the brain parenchyma [13], recent findings have revealed that they also participate in memory consolidation in the healthy brain by tweaking synapse connectivity and remodeling the extracellular matrix of the hippocampus upon experience-triggered secretion of interleukin-33 (IL-33) by neurons [14]. Further examples include IL-4-producing $\mathrm{CD}^{+} \alpha \beta \mathrm{T}$ cells and IL-17-producing $\gamma \delta$ T cells in the meninges that respectively contribute to spatial learning and short-term memory, just to name a few $[15,16]$.

However, upon inflammation, immune cells play their classic protective activities to eliminate a given stressor (e.g., antigenic overload or oxidative imbalance) and to restore tissue physiology. In a second vein, an inflamma- tion may become chronic when the stressor is not completely cleared. The persistence of pro-inflammatory molecules and the influx of immune cells may lead to tissue dysfunction and the emergence of disease, including autoimmune and neurodegenerative disorders. In this context, brain infiltration by blood-trafficking immune cells is regarded as a well-regulated process, with adhesive molecules expressed on the endothelium playing a major role, as compared to the proper barrier structure [17]. The integrity of the blood-brain barrier (BBB) also involves specialized tight junctions between endothelial cells and the close contact of their basement membranes with pericytes and the foot processes of astrocytes, thus limiting solute transport and cell entry to the brain. The trafficking of immune cells has been approached in several studies, including autoimmune and infectious diseases, as well as in noninfectious nonautoimmune conditions affecting the CNS. Particularly, this process has been reported comprehensively in several experimental and clinical studies searching for understanding the immunopathogenesis of multiple sclerosis (MS). This disease has a complex pathological pattern but is characterized by myelin sheath damage directed by inflammation, with specific immune cell infiltration contributing primarily for the disease onset, which may progress to neurodegeneration $[18,19]$. Notably, the studies with MS patients, along with the reports from the distinct experimental autoimmune encephalomyelitis (EAE) models, point to a concerted role for the main effector Th1 and Th17 CD4 ${ }^{+} \mathrm{T}$ cells [18]. The pathological basis for the neuroinflammation and active demyelination also involves cytolytic $\mathrm{CD} 8^{+} \mathrm{T}$ cell [20] and B cell [21] subsets in addition to the relevant infiltrating macrophages and activated resident microglia $[18,22]$, among other immune components.

In order to bind the brain endothelium and transmigrate into the CNS during MS evolution, the pathogenic immune cells engage in a multifaceted interactive process, which involves the exit from the bloodstream and the transient E- and P-selectin-mediated cell tethering and rolling over the activated endothelium [23]. This process is triggered by inflamed tissue-derived cytokines, including tumor necrosis factor and chemokines, with the latter driving integrin activation. This is an essential step preceding migration toward the injured site, as integrins show a bidirectional plasma membrane communication that regulates their structural activation via conformational alteration $[24,25]$. The outside-in signaling follows the direct integrin ligation and the inside-out signaling occurs via engagement of nonintegrin receptors, includ- 
ing chemokine-receptors, which signal intracellularly to a subsequent integrin conformational activation [23]. Importantly, a central role for the VLA-4 integrin has been demonstrated for such an active leukocyte migratory pathway [26]. Upon VLA-4 ligation, which follows the previous chemokine-driven integrin activation, the vascular cell adhesion molecule-1 (VCAM-1) operates the firm leukocyte adhesion on the activated endothelium. Following, the previously diffuse VCAM-1 distribution on the endothelial surface turns concentrated underneath the adherent/migrating VLA-4+ immune cell. The VLA-4/VCAM-1 interaction efficiently relays a Rac1-mediated signaling that destabilizes the endothelial junctional structures and promotes the consequent immune cell transmigration [27]. The BBB structure is also a critical controller for T-cell trafficking, as brain pericytes and astrocytes were reported to express VCAM-1, mediating their interaction with VLA- ${ }^{+} \mathrm{T}$ cells [28]. In addition, astrocytes seem to regionally regulate the T-cell trafficking as brain stem astrocyte express VCAM-1 in response to Th17 cytokines, whereas spinal cord astrocytes present the CXCL12/CXCR7 pathway as a driving cue in response to IFN- $\gamma$ [29]. Interestingly, VLA-4/ VCAM-1 pathway in T cell-astrocyte interaction might be related to the higher susceptibility of females to MS, since male sex hormones were able to suppress the expression of $\beta 1$ in female $\mathrm{T}$ cells primed with the myelin basic protein, whereas female sex hormone produced no effect [30].

In the CNS, the cognate autoantigen recognition by the pathogenic effector $\mathrm{CD} 4^{+} \mathrm{T}$ cells occurs via direct reactivation by antigen-presenting cells (APCs), with recruitment of additional effector T cells and macrophages, leading to the inflammatory lesions. Additionally, the VLA-4 integrin seems also to direct T cell/APC interactions within the CNS, representing an essential step for the neuroinflammation progress seen in MS [18]. In this regard, immature dendritic cells migrate into the CNS and further promote EAE by acting as professional APCs, an activity responsive to therapeutic blocking with anti- $\alpha 4$ integrin antibody [31]. Furthermore, a putative participation of B-cell-mediated $\mathrm{CD}^{+} \mathrm{T}$-cell activation in MS neuroinflammation was recently investigated by employing an EAE model and genetically approaching the antigen presentation function of the pathogenic B cells [32]. Through a conditional a 4 gene (Itga4) inactivation on B cells, it was suggested that VLA-4 is able to regulate the transmigration of B cells through VCAM- $1^{+}$activated endothelium and their subsequent meningeal clustering adjacently to cognate CD4 T cells [32].

Targeting VLA-4 in Neuroinflammation
These findings depict the fundamental role of VLA-4 in the complex steps leading to neuroinflammation and define this integrin as a molecular target for therapeutic blocking of the pathogenic migratory immune cells actively operating in the disease progression seen in MS patients. A broad structural basis for the VLA-4 targeting is discussed below, underscoring successful interventions on VLA-4-mediated immune cell migration to the CNS, both in the promising trials and in the currently successful therapy with the humanized monoclonal antibody natalizumab.

\section{Targeting the VLA-4 Molecule to Block Its Interactive Functions and the Basis for the Therapeutic Use in Neuroinflammatory Disorders}

Mostly, the specific recognition of VLA-4 by antibodies relies on the selective $\alpha 4$ subunit binding, since the $\beta 1$ subunit is shared with other integrins. In particular, the a4 subunit shows at least 3 major epitopes known as A, B, and $C$ [33]. The epitopes A and B are located at the $\beta$-propeller domain. Whereas the epitope A comprises the first 100 residues at the $\mathrm{N}$-terminus [33], the precise definition of epitope $\mathrm{B}$ is a little uncertain. By performing molecular mapping, it has been shown that epitope B can be further divided into epitope B1, encompassing residues 195-208, and epitope B2, comprising residues 108182 [34]. Other report defined the residues $152-203$ as epitope $\mathrm{B}$ without distinguishing between $\mathrm{B} 1$ and $\mathrm{B} 2$ regions [33]. The division of epitope $\mathrm{B}$ into 2 portions is supported by the finding that antibodies $\mathrm{H} 2 / 4$ or L25 binding to the $\mathrm{B} 2$ region induced homotypic cell aggregation, whereas anti-B1 antibodies HP1/2 or HP $2 / 1$ did not [35]. Clumping of cells was also stimulated by antibodies HP1/1, HP1/3, HP1/7, HP2/6, HP2/7, or MC3/2 anti-epitope $\mathrm{A}$, which inhibited VLA-4 interaction with fibronectin but not with VCAM-1 [35]. Indeed, subsequent mutagenesis studies have shown that asparagine residues 89 and 90 in the a 4 subunit were required for fibronectin interaction [36], thus suggesting another fibronectinbinding site besides MIDAS. Epitope $\mathrm{C}$ is also ill-defined, with some authors identifying it at residues 422-606, while others pointing to residues $269-548$ of the $a 4$ subunit thigh domain $[33,34]$. Due to its location away from the ligand-binding sites, antibodies B-5G10, B-5E2, or $8 \mathrm{~F} 2$ against epitope $\mathrm{C}$ did not impair VLA-4 interaction with VCAM-1 or fibronectin, nor promoted homotypic cell aggregation [35]. Currently, the anti-VLA-4 antibody mostly used in the clinic is natalizumab, which targets

Neuroimmunomodulation 2021;28:213-221 215 
epitope B in a noncompetitive way [37]. The development of this antibody was marked by the experimental evidence of a successful blocking activity for an antiVLA-4 antibody, which was able to inhibit the development of EAE [38]. This finding was followed by randomized, double-blind, and placebo-controlled clinical trials, which demonstrated the natalizumab efficacy in controlling inflammatory brain lesions and relapsing MS [3941].

Inhibition of VLA-4 can be achieved not only by antibodies but also by other approaches, such as small molecules and cyclic peptides, among others. In general, they are designed to exhibit higher affinity and stability in order to compete with and block endogenous integrin binding [42]. Of particular interest are the QIDSPL motif of VCAM-1 in the endothelial cells and the fibronectin LDV sequence $[43,44]$. Several small molecules have shown promising effects on preclinical assays and advanced to human clinical trials $[45,46]$. For instance, the $\alpha 4 \beta 1$ selective TBC3486 is a nonpeptide compound able to inhibit the binding to CS-1 fibronectin segment and VCAM1 , placing it as a critical target for modulation of T-cell migration through the BBB. This antagonist showed prominent results in the treatment of EAE mice, revealing a less severe acute phase, demyelination, and disease progression [47]. Also, the inhibitor BIO5192, designed from the LDV sequence, induced leukocytosis in the EAE model, which is an important marker of efficacy [48]. A structural optimization of VLA-4 antagonists leads to the small molecule 14e, which showed efficacy in experimental asthma and followed for phase I in clinical trials [49]. Nevertheless, the modification of molecules needs caution during lead compound derivation, since a simple change can modify its function. For instance, the THI0019 was the first VLA-4 small molecule agonist. It was generated by 2 structural modifications of TBC 3486 and was shown to improve rolling and spreading of the HPB-ALL cell line on VCAM-1 [50]. Other VLA-4 inhibitors were tested for different clinical approaches. BIO5192 was able to improve the mobilization of murine hematopoietic stem and progenitors, a critical step for the restoration of hematopoiesis after bone marrow transplantation [51]. Another inhibitor, BIO1211, exerted similar effect of corticosteroids in airway allergy response using a sheep model [52]. In addition, the antisense oligonucleotide inhibitor ATL1102 targets selectively the a 4 chain RNA, reducing VLA-4 expression and impairing cell adhesion. In a phase II trial, this drug decreased brain lesions in relapsing-remitting MS [53].

\section{Beyond the Therapeutic Blocking of VLA-4 Integrin}

The proper organization of cellular interactions correlates with the finely tuned cell signaling and the resulting functional activity, even during the transitory contacts in the immune response. As an example, integrins have been reported to coordinate phagocytosis through their regulatory role on generating actin rearrangements, which act as barrier to inhibitory phosphatases at the cellcell interface [54]. Particularly, the VLA-4 integrin-mediated interactions also relay critical signaling for proper immune cell function, including both the endothelialmediated T-cell migration and the APC-driven T-cell activation, as described above. Accordingly, any particular interference on these interactions might perturb relevant immune cell functions. In this sense, aberrant expression of integrins, as well as of other molecules directly involved in integrin-mediated interactions, has been associated with complex pathological conditions, including cancer and immunological diseases [55]. On the other hand, therapeutic targeting of integrins has been mechanistically explored and has successfully modified disease progression, including the neuroinflammation in MS [9].

In this perspective, one can envisage that targeting active integrin signaling on immune cells during their multistep migratory process, from the blood into the CNS, might also functionally modulate these neuroinflammatory cells. Yet, a comprehensive picture of a possible modulation of immune cell functions following VLA-4 targeting is still scarce.

\section{Functional Immune Modulation following VLA-4 Targeting}

Besides its role in the sequential adhesive and transmigratory activities in leukocytes, the integrin VLA-4 is able to mediate costimulatory function for T-cell activation [56]. This latter activity seems to be directly related to the presence of the integrin at the immunological synapse, colocalizing with the $\beta 2$-integrin LFA- 1 at the periphery of the T-cell contact area, the supra-molecular activation complex. Interestingly, this study showed the modulation of critical molecular and cellular functions when anti- $\alpha 4$ integrin antibodies were applied. Thus, the presence of anti- $\alpha 4$ alters VLA- 4 positioning at the T cell-APC interface, resulting in its co-localization with the $\mathrm{CD} 3-\zeta$ chain at the central region of supra-molecular activation complexes. Additionally, a polarized Th1 profile is achieved after the in vitro differentiation of naive $\mathrm{T}$ cells via the
Jurberg/Chaves/Pinho/da Silva/Savino/ Cotta-de-Almeida 
engagement of $\mathrm{CD} 3 / \mathrm{CD} 28 / \alpha 4$ integrin or via direct contact with mature dendritic cells in the presence of anti- $\alpha 4$ antibody. Similar Th1 polarization was observed following the in vivo anti- $\alpha 4$ treatment of a Th2-skewed autoimmune disease [56].

Interestingly, mice treated with $\beta 1$ integrin-activating antibody exhibited higher frequency of CD25 $5^{\text {high }}$ Treg cells, which showed increased suppressive activity [57]. Recent data also revealed that $\mathrm{T}$-cell devoid of $\alpha 4$ integrin (from conditional Itga4-deficient mice) had increased Foxp3 expression following in vitro alloreactive activation and showed lower expression of the T-bet, Gata3, and ROR $\gamma \mathrm{t}$ master transcription factors, as compared to control nonmutant $\mathrm{T}$ cells [58]. The possibly altered regulatory and effector functions might be added to the impaired migration ability of the a4-deficient $\mathrm{T}$ cells as the main mechanisms to explain the correlation of the VLA4 loss with the significant improvement in a mouse model of graft versus host disease [58].

Remarkably, mice with selective inactivation of the a4 integrin chain in T cells developed delayed EAE, with a higher frequency of CNS-infiltrating Th17 effector cells compared to Th1 lymphocytes. These and other findings suggest that VLA-4 has different effects on trafficking dynamics of distinct $\mathrm{T}$-cell subtypes or that Th17 cells may employ other integrins to reach the inflammatory site [59-61]. Moreover, EAE mice bearing a selective VLA-4 deficiency in B cells were shown to have lower disease susceptibility, possibly related to lower migratory activity of these B cells into the CNS [62]. Besides the scarcity of $B$ cells, these animals showed lower numbers of macrophages and $\mathrm{T}$ cells infiltrating the CNS. Interestingly, analysis of the infiltrating effector $\mathrm{T}$ cells revealed a significant reduction in the frequency of IL-17A-producing cells but not of IFN- $\gamma$-producing cells [62].

Considering that Treg cells are regarded as a highly VLA-4-expressing subset, it is predicted a possible regulatory role for this integrin on Treg cell interactive activities. Accordingly, adoptive transfer of Treg cells in EAE mice correlated with their enhanced migration to the peripheral lymph nodes and their high levels of adhesion molecules [63]. These events possibly facilitated functional interactions with the pathogenic $\mathrm{T}$ cells, resulting in the modulation of the Th1/Th2 balance along with diminished neuroinflammation and clinical improvement [63]. Therefore, therapy with anti- $\alpha 4$ antibodies might modulate specific T-cell effector functions by indirectly targeting Treg cells. The possibility of a more complex immune dysregulation effect deserves further investigation.

Targeting VLA-4 in Neuroinflammation
Another discrete modulatory impact by antibody targeting of VLA-4 might be attributed to its effect on mobilization of the human $\mathrm{CD} 34^{+}$hematopoietic progenitor cells, as observed in natalizumab-treated MS patients [64]. Since $\mathrm{CD}_{3} 4^{+}$cells are regarded as possessing immunoregulatory activity [65], the enhanced mobilizing activity of a synergistic combination of anti- $\alpha 4$ antibodies with granulocyte colony-stimulating factor is likely to provide further therapeutic value [66]. Interestingly, granulocyte colony-stimulating factor therapy has been shown to result in clinical improvement of patients with neuromuscular disorders, such as muscular dystrophies $[67,68]$. On the other hand, an attempt to restore the $\mathrm{BBB}$ in a preclinical model of stroke using transgenic mesenchymal stem cells overexpressing Itga4 to produce pericytes in the injured perivascular space showed no significant benefits in the repair of the BBB due to cell clearance [69].

These pivotal studies point that both gene targeting and antibody inhibition of VLA-4 on lymphocytes and mesenchymal stem cells modulate the neuroinflammation process. As VCAM-1-expressing brain pericytes and astrocytes seem to be additional cells able to interact with migrating inflammatory $\mathrm{T}$ cells $[28,29]$, therapeutic VLA-4 modulation might revert the leakage and restore the integrity of the BBB [70]. Importantly, this can be achieved not only by direct effect on adhesion blocking, but also by potentially regulating $\mathrm{T}$-cell differentiation and effector functions (Fig. 1).

\section{Anti-VLA-4 Therapy: Potential Application to Other Diseases and Its Undesirable Side Effects}

Given that VLA-4 holds a central role in the intricate immune cell adhesive and migratory events, which are key steps for the neuroinflammatory process, one might postulate the use of VLA-4 immunotherapy to slow down the progression of other neuromuscular and neurodegenerative disorders. In fact, considering that severity and rapid disease progression in Duchenne Muscular Dystrophy correlates with significantly increased levels of highly expressing VLA-4 T cells in the blood [71], the Duchenne Muscular Dystrophy patients could potentially benefit from the anti-VLA-4 treatment. Additionally, administration of a blocking anti-VLA-4 antibody to a transgenic mouse model of Alzheimer's disease resulted in mitigation of cognitive and neuropathological disorders associated to a reduced leukocyte migration and interaction with the brain vasculature [72].

Neuroimmunomodulation 2021;28:213-221 


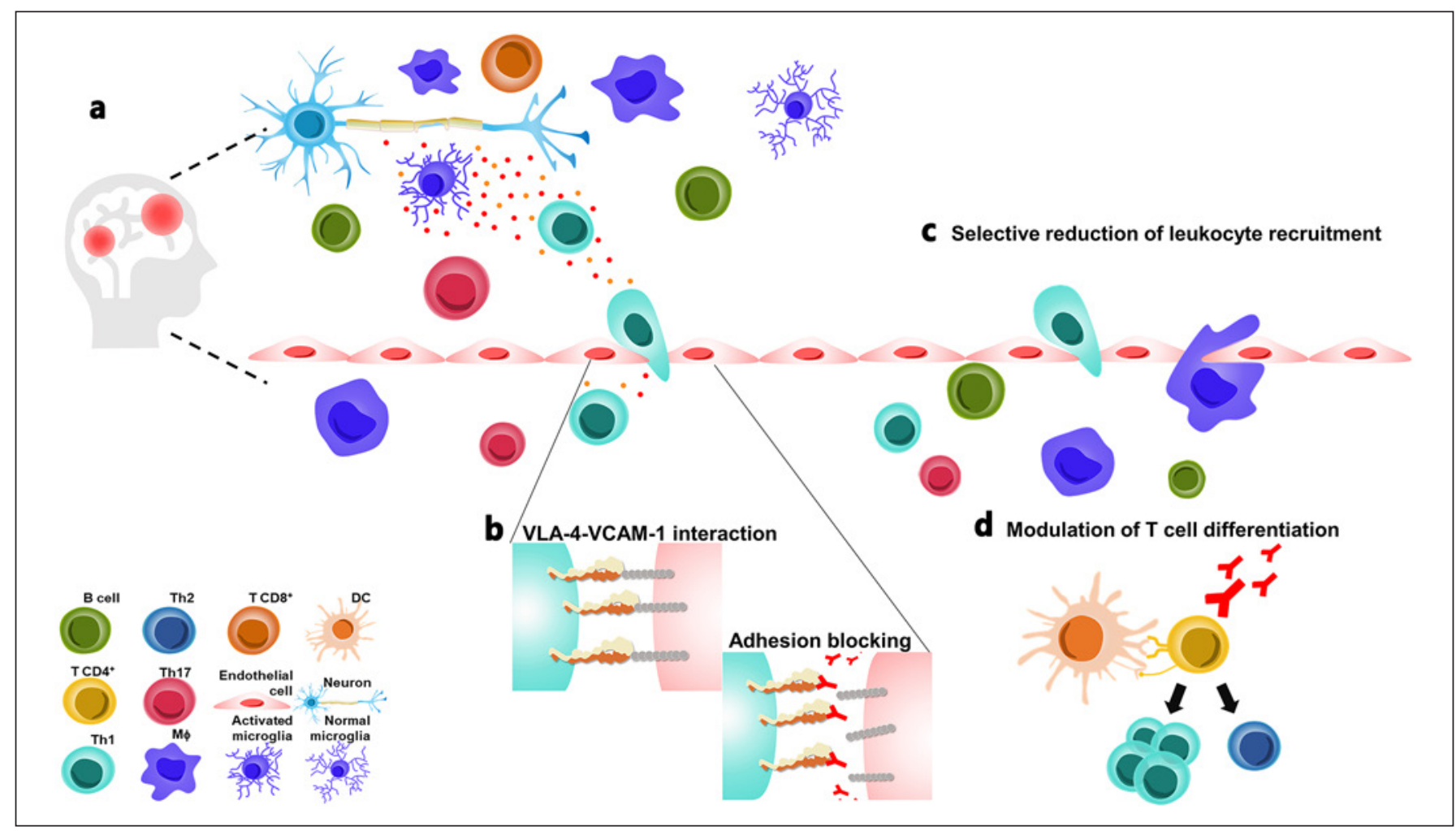

Fig. 1. Targeting of VLA-4 integrin might functionally modulate $\mathrm{T}$ cells in addition to blocking immune cell infiltration. a The myelin sheath breakdown, following the disruption of the BBB found in MS, involves a neuroinflammation course with infiltrating lymphocytes and macrophages, as well as activated resident microglia. b Central in this process is the VLA-4/VCAM-1-mediated interactions between leukocytes and endothelial cells, which promote the extravasation of immune cells into the CNS and are blocked by
anti-VLA-4 antibodies. c, d The insets depict the postulate that VLA-4 targeting might also modulate immune cell function. This can be denoted from studies that apply specific antibodies during T-cell activation/differentiation [56] and employ selective ITGA4 inactivation in both $\mathrm{B}$ cells [62] and $\mathrm{CD}^{+} \mathrm{T}$ cells [59]. CNS, central nervous system; BBB, blood-brain barrier; MS, multiple sclerosis; VCAM, vascular cell adhesion molecule.
However, despite the patent efficacy of the antiVLA-4 therapy in blocking the arrival of effector immune cells within inflammation sites and in reducing MS relapses, the natalizumab treatment is associated with the appearance of the opportunistic infection with the John Cunningham virus in the SNC [40]. A detrimental pathological process follows with the development of progressive multifocal leukoencephalitis illness. Interestingly, MS worsening has been also reported following other immunomodulatory treatments [18]. A more complex view on the distinct effects of VLA-4 immunotherapy for neuroinflammation considers the role of astrocytes as VCAM-1-expressing cells, providing the molecular cue for interacting with inflammatory VLA $-4^{+}$leukocytes. In this sense, despite the several studies showing that activated astrocytes contribute to the neuroinflammation, a protective role for these cells in regulating inflammation and supporting $\mathrm{BBB}$ has been debated $[73,74]$.

On the other hand, it is important to point out that natalizumab treatment withdrawal has been associated to severe rebound of MS relapses and enhanced presence of EBV-reactivated B cells in MS lesions $[75,76]$. Together, these observations conceptually point that more complex immune mechanisms might be operating to develop the undesirable immune effects elicited after anti-VLA-4 therapy.

\section{Concluding Remarks}

As reviewed herein, there seems to be a concomitant role for VLA-4 in the adhesion and migration interactions as well as in modulating some immune cell activi- 
ties, including T-cell activation and effector functions. Such a level of complexity may arise after the integrated signals from cross-talking membrane receptors that contribute to properly regulated T-cell functions. This can be exemplified by the combined activity of integrins and chemotactic receptors [77] and by the co-regulation of distinct integrins, as demonstrated that LFA-1 binding modulates the VLA-4 phosphorylation status [78]. Interestingly, anti-LFA-1 antibodies were shown to inhibit VLA-4 binding to VCAM-1 [79]. These mechanistic findings point that advanced anti-VLA-4 therapies might combine other immunomodulatory treatments.

Another aspect is related to new technological approaches regarding the antibody format and specificity. For example, we have recently produced an anti-VLA-4 single-chain variable fragment antibody with the ability to inhibit the adhesion of Jurkat cells on VCAM-1-coated surface (pending patent; Brazilian National Institute of Intellectual Property - INPI - number BR 102020016890 $8)$. This antibody fragment was developed from an in silico comparative modeling for selective binding to $\alpha 4 \beta 1$ but not to $\alpha 4 \beta 7$ integrin. Additional assays are still needed to evaluate whether this antibody has distinct effects on immune cell functions compared to natalizumab. A similar strategy on computational design can be envisaged for differential targeting of distinct T-cell subsets on the trafficking pathway for neuroinflammation. The modeling of a bi-specific antibody for concomitant binding to VLA-4 and a specific cell marker (e.g., Th1 vs. Th17) could be a challenging strategy.

Lastly, despite the long-time use of the VLA-4 antibody therapy, which emerged from the first observations on inhibition of in vivo leukocyte extravasation [80], the topics reviewed herein point that VLA-4 relevance on neuroinflammation still poses interesting challenges. In this context, the development of further effective immunomodulatory VLA-4-based therapies is warranted.

\section{Acknowledgments}

The authors thank the stimulating interactions and discussions around the topic leukocyte migration, immunotherapeutic intervention, and VLA-4 function with Drs. Lisa Westerberg, Loic Dupré, Marco Alberto Medeiros, and Zilton Vasconcelos. This work is dedicated to the memory of a dear friend and enthusiastic scientist, Dr. Juliana De Meis.

\section{Conflict of Interest Statement}

The authors declare no conflict of interest.

\section{Funding Sources}

This work was supported by the Oswaldo Cruz Institute (internal budget for the Laboratory on Thymus Research), Fiocruz (Inova Fiocruz Program - Generation of knowledge 2018-\#182104), CNPq (VCA supported by \#310329/2019-1), CAPES, FAPERJ, Estacio de Sá University (Productivity in Research Program) and the Mercosur Fund for Structural Convergence (FOCEM; \#03/11). It was developed in the frameworks of the Brazilian National Institute of Science and Technology on Neuroimmunomodulation and the Rio de Janeiro Research Network on Neuroinflammation.

\section{Author Contributions}

A.D.J., B.C., L.G.P., J.H.M.S., W.S., and V.C.-A. contributed for the review conceptualization and the writing process. All the authors revised critically and approved the final manuscript.

\section{References}

1 Kassebaum NJ, Arora M, Barber RM, Bhutta ZA, Brown J, Carter A, et al. Global, regional, and national disability-adjusted life-years (DALYs) for 315 diseases and injuries and healthy life expectancy (HALE), 1990-2015: a systematic analysis for the Global Burden of Disease Study 2015. Lancet. 2016 Oct; 388(10053):1603-58.

2 James SL, Abate D, Abate KH, Abay SM, Abbafati C, Abbasi N, et al. Global, regional, and national incidence, prevalence, and years lived with disability for 354 diseases and injuries for 195 countries and territories, 19902017: a systematic analysis for the Global Burden of Disease Study 2017. Lancet. 2018; 392(10159):1789-858.
3 Driver JA, Logroscino G, Gaziano JM, Kurth $\mathrm{T}$. Incidence and remaining lifetime risk of Parkinson disease in advanced age. Neurology. 2009;72(5):432-8.

4 Ransohoff RM. How neuroinflammation contributes to neurodegeneration. Science. 2016;353(6301):777-83.

5 Franceschi C, Garagnani P, Parini P, Giuliani C, Santoro A. Inflammaging: a new immunemetabolic viewpoint for age-related diseases. Nat Rev Endocrinol. 2018;14(10):576-90.

6 McHugh D, Gil J. Senescence and aging: causes, consequences, and therapeutic avenues. J Cell Biol. 2018;217(1):65-77.
7 Ahadi S, Zhou W, Schüssler-Fiorenza Rose SM, Sailani MR, Contrepois K, Avina M, et al. Personal aging markers and ageotypes revealed by deep longitudinal profiling. Nat Med. 2020;26(1):83-90.

8 Springer TA. Traffic signals for lymphocyte recirculation and leukocyte emigration: the multistep paradigm. Cell. 1994 Jan;76(2): 301-14.

9 Ley K, Rivera-Nieves J, Sandborn WJ, Shattil S. Integrin-based therapeutics: biological basis, clinical use and new drugs. Nat Rev Drug Discov. 2016 Mar;15(3):173-83.

10 Ransohoff RM, Engelhardt B. The anatomical and cellular basis of immune surveillance in the central nervous system. Nat Rev Immunol. 2012;12(9):623-35. 
11 Korn T, Kallies A. T cell responses in the central nervous system. Nat Rev Immunol. 2017; 17(3):179-94.

12 Prinz M, Priller J. The role of peripheral immune cells in the CNS in steady state and disease. Nat Neurosci. 2017 Feb;20(2):136-44.

13 Thameem Dheen S, Kaur C, Ling E-A. Microglial activation and its implications in the brain diseases. Curr Med Chem. 2007;14(11): 1189-97.

14 Nguyen PT, Dorman LC, Pan S, Vainchtein ID, Han RT, Nakao-Inoue H, et al. Microglial remodeling of the extracellular matrix promotes synapse plasticity. Cell. 2020;182(2): 388-403.e15.

15 Derecki NC, Cardani AN, Yang CH, Quinnies KM, Crihfield A, Lynch KR, et al. Regulation of learning and memory by meningeal immunity: a key role for IL-4. J Exp Med. 2010; 207(5):1067-80.

16 Ribeiro M, Brigas HC, Temido-Ferreira M, Pousinha PA, Regen T, Santa C, et al. Meningeal $\gamma \delta$ T cell-derived IL-17 controls synaptic plasticity and short-term memory. Sci Immunol. 2019;4(40):eaay5199.

17 Greenwood J, Heasman SJ, Alvarez JI, Prat A, Lyck R, Engelhardt B. Review: leucocyte-endothelial cell crosstalk at the blood-brain barrier: a prerequisite for successful immune cell entry to the brain. Neuropathol Appl Neurobiol. 2011;37(1):24-39.

18 Baecher-Allan C, Kaskow BJ, Weiner HL Multiple sclerosis: mechanisms and immunotherapy. Neuron. 2018;97(4):742-68.

19 McFarland HF, Martin R. Multiple sclerosis: a complicated picture of autoimmunity. Nat Immunol. 2007;8(9):913-9.

20 Martin-Blondel G, Pignolet B, Tietz S, Yshii L, Gebauer C, Perinat T, et al. Migration of encephalitogenic CD8 T cells into the central nervous system is dependent on the $\alpha 4 \beta 1$ integrin. Eur J Immunol. 2015;45(12):330212.

21 Molnarfi N, Schulze-Topphoff U, Weber MS, Patarroyo JC, Prod'homme T, Varrin-Doyer $\mathrm{M}$, et al. MHC class II-dependent B cell APC function is required for induction of CNS autoimmunity independent of myelin-specific antibodies. J Exp Med. 2013 Dec;210(13): 2921-37.

22 Lucchinetti CF, Popescu BF, Bunyan RF, Moll $\mathrm{NM}$, Roemer SF, Lassmann $\mathrm{H}$, et al. Inflammatory cortical demyelination in early multiple sclerosis. N Engl J Med. 2011;365(23): 2188-97.

23 Luo BH, Carman CV, Springer TA. Structural basis of integrin regulation and signaling. Annu Rev Immunol. 2007 Apr;25(1):619-47.

24 Campbell ID, Humphries MJ. Integrin structure, activation, and interactions. Cold Spring Harb Perspect Biol. 2011;3(3):1-14

25 Chigaev A, Sklar LA. Aspects of VLA-4 and LFA-1 regulation that may contribute to rolling and firm adhesion. Front Immunol. 2012; 3:242.
26 van Langelaar J, Rijvers L, Smolders J, van Luijn MM. B and T cells driving multiple sclerosis: identity, mechanisms and potential triggers. Front Immunol. 2020;11:760.

27 Muller WA. Localized signals that regulate transendothelial migration. Curr Opin Immunol. 2016 Feb;38(3):24-9.

28 Gimenez MA, Sim JE, Russell JH. TNFR1-dependent VCAM-1 expression by astrocytes exposes the CNS to destructive inflammation. J Neuroimmunol. 2004;151(1-2):116-25.

29 Williams JL, Manivasagam S, Smith BC, Sim J, Vollmer LL, Daniels BP, et al. Astrocyte-T cell crosstalk regulates region-specific neuroinflammation. Glia. 2020;68(7):1361-74.

30 Brahmachari S, Pahan K. Gender-specific expression of $\beta 1$ integrin of VLA- 4 in myelin basic protein-primed $T$ cells: implications for gender bias in multiple sclerosis. J Immunol. 2010;184(11):6103-13.

31 Jain P, Coisne C, Enzmann G, Rottapel R, Engelhardt B. Alpha4betal integrin mediates the recruitment of immature dendritic cells across the blood-brain barrier during experimental autoimmune encephalomyelitis. J Immunol. 2010 Jun;184(12):7196-206.

32 Parker Harp CR, Archambault AS, Cheung M, Williams JW, Czepielewski RS, Duncker PC, et al. Neutrophils promote VLA-4-dependent $\mathrm{B}$ cell antigen presentation and accumulation within the meninges during neuroinflammation. Proc Natl Acad Sci U S A. 2019;116(48):24221-30.

33 Schiffer SG, Hemler ME, Lobb RR, Tizard R, Osborn L, Hemler ME, et al. Molecular mapping of functional antibody binding sites of alpha 4 integrin. J Biol Chem. 1995 Jun; 270(24):14270-3.

34 Kamata T, Puzon W, Takada Y. Identification of putative ligand-binding sites of the integrin a4ß1 (VLA-4, CD49d/CD29). Biochem J. 1995 Feb;305(3):945-51.

35 Pulido R, Elices MJ, Campanero MR, Osborn L, Schiffer S, Garcia-Pardo A, et al. Functional evidence for three distinct and independently inhibitable adhesion activities mediated by the human integrin VLA-4. Correlation with distinct $\alpha 4$ epitopes. J Biol Chem. 1991; 266(16):10241-5.

36 Munoz M, Serrador J, Sanchez-Madrid F, Teixido J. A region of the integrin VLA 4 subunit involved in homotypic cell aggregation and in fibronectin but not vascular cell adhesion molecule-1 binding. J Biol Chem. 1996 Feb;271(5):2696-702.

$37 \mathrm{Yu}$ Y, Schürpf T, Springer TA. How natalizumab binds and antagonizes a4 integrins. J Biol Chem. 2013 Nov;288(45):32314-25.

38 Yednock TA, Cannon C, Fritz LC, SanchezMadrid F, Steinman L, Karin N. Prevention of experimental autoimmune encephalomyelitis by antibodies against alpha 4 beta 1 integrin. Nature. 1992 Mar;356(6364):63-6.
39 Miller DH, Khan OA, Sheremata WA, Blumhardt LD, Rice GP, Libonati MA, et al. A controlled trial of natalizumab for relapsing multiple sclerosis. N Engl J Med. 2003 Jan;348(1): 15-23.

40 Polman $\mathrm{CH}$, O'Connor PW, Havrdova E, Hutchinson M, Kappos L, Miller DH, et al. A randomized, placebo-controlled trial of natalizumab for relapsing multiple sclerosis. $\mathrm{N}$ Engl J Med. 2006 Mar;354(9):899-910.

41 Tubridy N, Behan PO, Capildeo R, Chaudhuri A, Forbes R, Hawkins CP, et al. The effect of anti-alpha4 integrin antibody on brain lesion activity in MS. The UK Antegren Study Group. Neurology. 1999 Aug;53(3):466-72.

42 Millard M, Odde S, Neamati N. Integrin targeted therapeutics. Theranostics. 2011;1:15488.

43 Komoriya A, Green LJ, Mervic M, Yamada SS, Yamada KM, Humphries MJ. The minimal essential sequence for a major cell type-specific adhesion site (CS1) within the alternatively spliced type III connecting segment domain of fibronectin is leucine-aspartic acid-valine. J Biol Chem. 1991;266(23):15075-9.

44 Kilger G, Needham LA, Nielsen PJ, Clements J, Vestweber D, Holzmann B. Differential regulation of alpha 4 integrin-dependent binding to domains 1 and 4 of vascular cell adhesion molecule-1. J Biol Chem. 1995 Mar;270(11): 5979-84.

45 Rettig MP, Ansstas G, Dipersio JF. Mobilization of hematopoietic stem and progenitor cells using inhibitors of CXCR4 and VLA-4. Leukemia. 2012;26(1):34-53.

46 ClinicalTrials.gov [Internet]. [cited 2020 Aug 28]. Available from: https://clinicaltrials.gov/ ct $2 /$ results? cond $=\&$ term $=$ integrin $\&$ cntry $=\&$ state $=\&$ city $=\&$ dist $=$.

47 Cannella B, Gaupp S, Tilton RG, Raine CS Differential efficacy of a synthetic antagonist of VLA-4 during the course of chronic relapsing experimental autoimmune encephalomyelitis. J Neurosci Res. 2003;71(3):407-16.

48 Leone DR, Giza K, Gill A, Dolinski BM, Yang $\mathrm{W}$, Perper S, et al. An assessment of the mechanistic differences between two integrin alpha 4 beta 1 inhibitors, the monoclonal antibody TA-2 and the small molecule BIO5192, in rat experimental autoimmune encephalomyelitis. J Pharmacol Exp Ther. 2003;305(3):115062.

49 Muro F, Iimura S, Sugimoto Y, Yoneda Y, Chiba J, Watanabe T, et al. Discovery of trans4-[1-[[2,5-dichloro-4-(1-methyl-3-indolylcarboxamido) phenyl]acetyl]-(4S)-methoxy(2S)-pyrrolidinylmethoxy]cyclohexanecarboxylic acid: an orally active, selective very late antigen-4 antagonist. J Med Chem. 2009; 52(24):7974-92.

50 Vanderslice P, Biediger RJ, Woodside DG, Brown WS, Khounlo S, Warier ND, et al. Small molecule agonist of very late antigen-4 (VLA-4) integrin induces progenitor cell adhesion. J Biol Chem. 2013;288(27):19414-28. 
51 Ramirez P, Rettig MP, Uy GL, Deych E, Holt MS, Ritchey JK, et al. BIO5192, a small molecule inhibitor of VLA-4, mobilizes hematopoietic stem and progenitor cells. Blood. 2009;114(7):1340-3.

52 Lin KC, Ateeq HS, Hsiung SH, Chong LT, Zimmerman CN, Castro A, et al. Selective, tight-binding inhibitors of integrin alpha4beta1 that inhibit allergic airway responses. J Med Chem. 1999;42(5):920-34.

53 Limmroth V, Barkhof F, Desem N, Diamond MP, Tachas G; ATL1102 Study Group. CD49d antisense drug ATL1102 reduces disease activity in patients with relapsing-remitting MS. Neurology. 2014;83(20):1780-8.

54 Freeman SA, Goyette J, Furuya W, Woods EC, Bertozzi CR, Bergmeier W, et al. Integrins form an expanding diffusional barrier that coordinates phagocytosis. Cell. 2016;164(1-2): $128-40$.

55 Winograd-Katz SE, Fässler R, Geiger B, Legate KR. The integrin adhesome: from genes and proteins to human disease. Nat Rev Mol Cell Biol. 2014 Apr;15(4):273-88.

56 Mittelbrunn M, Molina A, Escribese MM, Yáñez-Mó M, Escudero E, Ursa A, et al. VLA4 integrin concentrates at the peripheral supramolecular activation complex of the immune synapse and drives T helper 1 responses. Proc Natl Acad Sci U S A. 2004;101(30): 11058-63.

57 Klann JE, Kim SH, Remedios KA, He Z, Metz PJ, Lopez J, et al. Integrin activation controls regulatory $\mathrm{T}$ cell-mediated peripheral tolerance. J Immunol. 2018;200(12):4012-23.

58 Alahmari B, Cooper ML, Vij K, Ritchey J, Ruminski P, Gao F, et al. Selective targeting of $\alpha 4 \beta 1$ integrin attenuates murine graft versus host disease. Leukemia. 2020;34(11):3100-4.

59 Glatigny S, Duhen R, Oukka M, Bettelli E. Cutting edge: loss of $a 4$ integrin expression differentially affects the homing of Th1 and Th17 cells. J Immunol. 2011;187(12):6176-9.

60 Sprenger H, Beck J, Nain M, Wesemann W, Gemsa D. Th17 lymphocytes traffic to the central nervous system independently of $a 4$ integrin expression during EAE. Immunobiology. 1991 Sep;183(1-2):94-101.

61 Sprenger H, Beck J, Nain M, Wesemann W, Gemsa D. LFA-1 controls Th1 and Th17 motility behavior in the inflamed central nervous system. Immunobiology. 1991 Sep;183(1-2): 94-101.
62 Lehmann-Horn K, Sagan SA, Bernard CC, Sobel RA, Zamvil SS. B-cell very late antigen-4 deficiency reduces leukocyte recruitment and susceptibility to central nervous system autoimmunity. Ann Neurol. 2015;77(5):902-8.

63 Kohm AP, Carpentier PA, Anger HA, Miller SD. Cutting edge: $\mathrm{CD} 4+\mathrm{CD} 25+$ regulatory $\mathrm{T}$ cells suppress antigen-specific autoreactive immune responses and central nervous system inflammation during active experimental autoimmune encephalomyelitis. J Immunol. 2002 Nov; 169(9):4712-6.

64 Zohren F, Toutzaris D, Klärner V, Hartung HP, Kieseier B, Haas R. The monoclonal antiVLA-4 antibody natalizumab mobilizes CD34+ hematopoietic progenitor cells in humans. Blood. 2008 Apr;111(7):3893-5.

65 Gur H, Krauthgamer R, Bachar-Lustig E, Katchman H, Arbel-Goren R, Berrebi A, et al. Immune regulatory activity of CD34+ progenitor cells: evidence for a deletion-based mechanism mediated by TNF- $\alpha$. Blood. 2005; 105(6):2585-93.

66 Christ O, Kronenwett R, Haas R, Zöller M. Combining G-CSF with a blockade of adhesion strongly improves the reconstitutive capacity of mobilized hematopoietic progenitor cells. Exp Hematol. 2001;29(3):380-90.

67 Eljaszewicz A, Sienkiewicz D, Grubczak K, Okurowska-Zawada B, Paszko-Patej G, Miklasz $\mathrm{P}$, et al. Effect of periodic granulocyte colony-stimulating factor administration on endothelial progenitor cells and different monocyte subsets in pediatric patients with muscular dystrophies. Stem Cells Int. 2015; 2016:2650849.

68 Sienkiewicz D, Kułak W, Okurowska-Zawada B, Paszko-Patej G, Wojtkowski J, Sochoń K, et al. Efficacy and the safety of granulocyte colony-stimulating factor treatment in patients with muscular dystrophy: a non-randomized clinical trial. Front Neurol. 2017 Oct;8:566.

69 Andrzejewska A, Dabrowska S, Nowak B, Walczak P, Lukomska B, Janowski M. Mesenchymal stem cells injected into carotid artery to target focal brain injury home to perivascular space. Theranostics. 2020;10(15):6615-28.

70 Soon D, Altmann DR, Fernando KT, Giovannoni G, Barkhof F, Polman CH, et al. A study of subtle blood brain barrier disruption in a placebo-controlled trial of natalizumab in relapsing remitting multiple sclerosis. J Neurol. 2007;254(3):306-14.
71 Sprenger H, Beck J, Nain M, Wesemann W, Gemsa D. CD49d is a disease progression biomarker and a potential target for immunotherapy in Duchenne muscular dystrophy. Immunobiology. 1991 Sep;183(1-2):94-101.

72 Pietronigro E, Zenaro E, Bianca VD, Dusi S, Terrabuio E, Iannoto G, et al. Blockade of $\alpha 4$ integrins reduces leukocyte-endothelial interactions in cerebral vessels and improves memory in a mouse model of Alzheimer's disease. Sci Rep. 2019;9(1):12055-15.

73 Sofroniew MV. Astrocyte barriers to neurotoxic inflammation. Nat Rev Neurosci. 2015; 16(5):249-63.

74 Lopes Pinheiro MA, Kooij G, Mizee MR, Kamermans A, Enzmann G, Lyck R, et al. Immune cell trafficking across the barriers of the central nervous system in multiple sclerosis and stroke. Biochim Biophys Acta. 2016; 1862(3):461-71.

75 Serafini B, Scorsi E, Rosicarelli B, Rigau V, Thouvenot E, Aloisi F. Massive intracerebral Epstein-Barr virus reactivation in lethal multiple sclerosis relapse after natalizumab withdrawal. J Neuroimmunol. 2017 Jun;307:14-7.

76 Sorensen PS, Koch-Henriksen N, Petersen T, Ravnborg M, Oturai A, Sellebjerg F. Recurrence or rebound of clinical relapses after discontinuation of natalizumab therapy in highly active MS patients. J Neurol. 2014 Jun; 261(6):1170-7.

77 Vicente-Manzanares M, Cruz-Adalia A, Martín-Cófreces NB, Cabrero JR, Dosil M, Alvarado-Sánchez B, et al. Control of lymphocyte shape and the chemotactic response by the GTP exchange factor Vav. Blood. 2005; 105(8):3026-34.

78 Uotila LM, Jahan F, Soto Hinojosa L, Melandri E, Grönholm M, Gahmberg CG. Specific phosphorylations transmit signals from leukocyte $\beta 2$ to $\beta 1$ integrins and regulate adhesion. J Biol Chem. 2014;289(46):32230-42.

79 Grönholm M, Jahan F, Bryushkova EA, Madhavan S, Aglialoro F, Soto Hinojosa L, et al. LFA-1 integrin antibodies inhibit leukocyte a4 $\beta 1$-mediated adhesion by intracellular signaling. Blood. 2016;128(9):1270-81.

80 Issekutz TB. Inhibition of in vivo lymphocyte migration to inflammation and homing to lymphoid tissues by the TA-2 monoclonal antibody. A likely role for VLA-4 in vivo. J Immunol. 1991 Dec;147(12):4178-84. 